\title{
Antitumor Effect of Periplocin in TRAIL-Resistant Human Hepatocellular Carcinoma Cells through Downregulation of IAPs
}

\author{
Chieh-Fang Cheng, I-Huang Lu, Hsiang-Wen Tseng, Chung-Yuan Sun, Li-Tsen Lin, \\ Zong-Keng Kuo, I-Horng Pan, and Ching-Huai Ko
}

Biomedical Technology and Device Research Laboratories, Industrial Technology Research Institute, Hsinchu, Taiwan

Correspondence should be addressed to I-Horng Pan; i-horngpan@itri.org.tw and Ching-Huai Ko; chko@itri.org.tw

Received 23 August 2012; Revised 15 November 2012; Accepted 15 November 2012

Academic Editor: Andreas Sandner-Kiesling

Copyright @ 2013 Chieh-Fang Cheng et al. This is an open access article distributed under the Creative Commons Attribution License, which permits unrestricted use, distribution, and reproduction in any medium, provided the original work is properly cited.

\begin{abstract}
Cortex periplocae is the dried root bark of Periploca sepium Bge., a traditional Chinese herb medicine. It contains high amounts of cardiac glycosides. Several cardiac glycosides have been reported to inhibit tumor growth or induce tumor cell apoptosis. We extracted and purified cortex periplocae and identified periplocin as the active ingredient that inhibited the growth of TNFrelated apoptosis-inducing ligand-(TRAIL-) resistant hepatocellular carcinoma cells. The antitumor activity of periplocin was further increased by TRAIL cotreatment. Periplocin sensitized TRAIL-resistant HCC through the following two mechanisms. First, periplocin induced the expression of DR4 and FADD. Second, the cotreatment of TRAIL and periplocin suppressed several inhibitors of apoptosis (IAPs). Both mechanisms resulted in the activation of caspase 3, 8, and 9 and led to cell apoptosis. In addition, intraperitoneal injection (IP) of periplocin repressed the growth of hepatocellular carcinoma (HCC) in xenograft tumor model in mice. In summary, periplocin sensitized TRAIL-resistant HCC cells to TRAIL treatment and resulted in tumor cell apoptosis and the repression of tumor growth in vivo.
\end{abstract}

\section{Introduction}

Liver cancer is the fifth leading malignancy in men and the ninth in women worldwide [1]. The incidence of liver cancer is highly correlated to chronic local inflammation and cirrhosis. Therefore, factors that stimulate inflammation in liver, including alcohol uses, infection of hepatitis B and C viruses, and fatty liver diseases, are strongly correlated to the pathological progression of liver cancer.

Current treatments for liver cancer are limited. Hepatectomy can be used in early stage liver cancer patients with functional liver. Liver transplantation can help patients with damaged livers, but matching suitable donors is not easy. Although these surgical operations work well in early stage diseases, they are not helpful for patients with cancer cells spread out of the liver. Chemotherapy and internal radiation therapy are also options for liver cancer treatment. However, they may do damage to other tissues and organs as well. Targeted therapy is a more specific treatment for cancer. Sorafenib, a multireceptor kinase inhibitor with antiangiogenic activity, is the standard treatment for advanced hepatocellular carcinoma (HCC) that cannot be removed with surgery. Although it extends the median overall survival in patients with advanced HCC for nearly 3 months, sorafenib does not increase the median time to symptomatic progression in patients with advanced HCC [2]. Therefore, novel treatments for HCC are strongly in need.

With the advancement of techniques in extraction, isolation, and recognition of compounds from plants, scientists started to search for antitumor components from herb medicine [3-6]. Cortex periplocae is the dried root bark of Periploca sepium Bge. It contains several cardiac glycosides and can be used in the treatment of various heart conditions. Recent studies also suggest that periplocin, a cardiac glycoside extracted from cortex periplocae, can inhibit cell growth in colon cancer cells and lung cancer cells $[7,8]$.

TNF-related apoptosis-inducing ligand (TRAIL) is a member of the tumor necrosis factor superfamily. It is also known as CD253 and APO-2L. TRAIL binds to the death 
receptors DR4 and DR5 and induces cell apoptosis [911]. Therefore, TRAIL is a potential candidate for cancer treatment [12]. In addition, phases 1 and 2 clinical trials for specific monoclonal antibodies against DR4 and DR5 have provided promising results [13].

Although TRAIL is a promising chemotherapeutic target for cancers, resistance to TRAIL-induced apoptosis has been reported in several different cancers, including colorectal cancer, breast cancer, liver cancer, and pancreatic cancer [1417]. Several different mechanisms are proposed for TRAIL resistance [18]. Ways to overcome TRAIL resistance are still under investigation $[19,20]$. We sought to investigate the effect of periplocin in sensitizing TRAIL-resistant HCC cell lines in this study.

\section{Material and Methods}

2.1. Cell Culture. HCC cell lines were purchased from different organizations. HA22T/VGH and Huh-7 were purchased from Bioresource Collection and Research Center (BCRC) in Taiwan. Huh-7 was purchased from Japanese Collection of Research Bioresources (JCRB). HA22T/VGH and Huh-7 were culture in DMEM (Gibco, Carlsbad, CA, USA) with 10\% FBS and $100 \mathrm{mM}$ nonessential amino acids (Gibco, Carlsbad, CA, USA).

2.2. Reagents. Recombinant human soluble TRAIL/APO2 ligand was purchased from ProSpec (Tany TechnoGene Ltd., Israel). Z-DEVD-FMK (CASP3 inhibitor), Z-IETD-FMK (CASP8 inhibitor), Z-LEHD-FMK (CASP9 inhibitor), and Z-VAD-FMK (pan CASP inhibitor) were purchased from $\mathrm{R}$ and $\mathrm{D}$ (Minneapolis, MN, USA). Monoclonal antihuman TRAIL R1 (TNFRSF10A,DR4)-Phycoerythrin antibody, antihuman TRAIL R3 (TNFRSF10C, DcR1)-Phycoerythrin antibody, and antihuman TRAIL R4 (TNFRSF10D, DcR2)Phycoerythrin antibody were purchased from $\mathrm{R}$ and D (Minneapolis, MN, USA). PE antihuman TRAILR2 (TNFRSF10B, DR5) antibody was purchased from Biolegend. (San Diego, CA, USA) N-acetyl-cysteine (NAC) and DCHFDA were purchased from Sigma Chemical Co. (St. Louis, MO, USA). Hydrogen peroxide $\left(\mathrm{H}_{2} \mathrm{O}_{2}\right)$ was purchased from MERCK (Whitehouse Station, NJ, USA).

2.3. Western Blot. Total cellular lysates were prepared by using RIPA lysis buffer. Proteins in cell lysates $(50 \mu \mathrm{g})$ were separated on 4-12\% SDS-polyacrylamide minigels and electrotransferred to a PVDF membrane by iBlot Dry Blotting System (Invitrogen, Carlsbad, CA, USA). Antibodies used in this study were as follows: anti-Caspase 3, 8, and 9 antibodies (Cell Signaling Technology, Boston, MA, USA; 1 : 2000), antiPARP antibody (Cell Signaling Technology, Boston, MA, USA; $1: 2000$ ), anti-BID antibody (Cell Signaling Technology, Boston, MA, USA; $1: 2000$ ), anti-FADD antibody (Cell Signaling Technology, Boston, MA, USA; 1 : 2000), anti-FLIP antibody (Cell Signaling Technology, Boston, MA, USA; $1: 2000$ ), antisurvivin antibody (Cell Signaling Technology, Boston, MA, USA; $1: 2000$ ), anti-Smac/Diablo antibody (Cell
Signaling Technology, Boston, MA, USA; 1:10000), antiBad antibody (Santa Cruz Biotechnology, Santa Cruz, CA, USA; 1 : 1000), anti-Bax antibody (Santa Cruz Biotechnology, Santa Cruz, CA, USA; $1: 1000$ ), anti-DR4 antibody (Santa Cruz Biotechnology, Santa Cruz, CA, USA; 1:1000), anti$\alpha$-tubulin antibody (Santa Cruz Biotechnology, Santa Cruz, CA, USA; 1:20000), anti-DR5 antibody (ProSci Incorporated, San Diego, California, USA; 1:500), anti-Bcl-2 antibody (BD Biosciences PharMingen, San Diego, CA, USA; $1: 1000$ ), anti-XIAP antibody (BD Biosciences PharMingen, San Diego, CA, USA; $1: 1000$ ), anti-cIAP-1 antibody (R and D Systems, Minneapolis, MN, USA; 1:1000), anti-cIAP2 antibody ( $\mathrm{R}$ and $\mathrm{D}$ Systems, Minneapolis, MN, USA; $1: 1000)$, anti-Mcl-1 antibody (Neomarker, Fremont, CA, USA; $1: 1000$ ), anti-Apaf-1 antibody (IMGENEX Corporation, San Diego, CA, USA; $1: 2000)$, and anti- $\beta$-Actin antibody (Novus Biologicals Inc, Colorado, USA; 1 : 10000).

2.4. Viability Assay (MTT). Cells were seeded at $10^{4}$ cells in $100 \mathrm{uL}$ medium per well in a 96-well plate and incubated $\left(37^{\circ} \mathrm{C}, 5 \% \mathrm{CO}_{2}\right)$ overnight. Drugs of interest were added to each well and incubated $\left(37^{\circ} \mathrm{C}, 5 \% \mathrm{CO}_{2}\right)$ for 2 days. MTT solution $(5 \mathrm{mg} / \mathrm{mL}$, Sigma Chemical Co., St. Louis, MO, USA) was added to each well at a final concentration of $0.5 \mathrm{mg} / \mathrm{mL}$ and incubated $\left(37^{\circ} \mathrm{C}, 5 \% \mathrm{CO}_{2}\right)$ for $1-2$ hours. Afterward, $100 \mathrm{uL}$ of $10 \%$ SDS (Fluka, St. Louis, MO, USA) was added to each well and incubated at room temperature overnight.

Optical density at $570 \mathrm{~nm}$ was detected by SpectraMax M5 (Molecular Devices, Sunnyvale, CA, USA) for quantification.

2.5. Determination of ROS Production. ROS production was monitored by flow cytometry using DCFH-DA. This dye is a stable compound that readily diffuses into cells and is hydrolyzed by intracellular esterase to yield DCFH, which is trapped within cells. Thus, the fluorescence intensity is detected to quantify the amount of peroxide produced by the cells. To investigate the effect of periplocin and the combination of periplocin and TRAIL on generating intracellular ROS in HA22T/VGH, cells were pretreated with $\mathrm{N}$-acetyl-cysteine (NAC) $(30 \mathrm{mM})$ for $30 \mathrm{~min}$ and followed by periplocin $(0.3$, $0.03 \mu \mathrm{M})$ alone or together with TRAIL (100 ng/mL). DCHFDA $(100 \mathrm{uM})$ were added to periplocin-treated cells with or without $\mathrm{H}_{2} \mathrm{O}_{2}(200 \mathrm{uM})$ for 1-2 hr. Green fluorescence was excited using an argon laser by flow cytometric analysis [21].

2.6. Quantification of Apoptosis by Annexin V/PI. After treated with periplocin alone or together with TRAIL for 24 hours, HA22T/VGH cells were washed and resuspended in the staining buffer and examined with the Vybrant Apoptosis Assay Kit (Invitrogen, Carlsbad, CA, USA) according to the manufacturer's instructions. The cell suspension was incubated with $2.5 \mu \mathrm{L}$ of Annexin $\mathrm{V}$ and $1 \mu \mathrm{L}$ of propidium iodide at room temperature for $15 \mathrm{~min}$. The stained cells were analyzed by fluorescence activated cell sorter (FACS) analyses with a FACSCalibur flow cytometer, and data were 
analyzed using CellQuest software (BD Biosciences, San Jose, CA, USA).

2.7. Quantification of Apoptosis by Sub-G1 Peak. Trypsinized cells were washed with ice-cold PBS and fixed in 70\% ethanol at $-20^{\circ} \mathrm{C}$ for at least $1 \mathrm{~h}$. After fixation, cells were washed twice, incubated in $0.5 \mathrm{~mL}$ of $0.5 \%$ Triton X-100/PBS at $37^{\circ} \mathrm{C}$ for $30 \mathrm{~min}$ with $1 \mathrm{mg} / \mathrm{mL}$ of RNase A, and stained with $0.5 \mathrm{~mL}$ of $50 \mathrm{mg} / \mathrm{mL}$ propidium iodide for $10 \mathrm{~min}$. The fluorescence emitted from the propidium-DNA complex was quantitated by FACSCalibur flow cytometer (BD Biosciences, San Jose, CA, USA).

2.8. FACS Analysis. Cells were incubated with dye-labeled monoclonal antibodies $(\mathrm{mAb})$ against target molecules for $30 \mathrm{~min}$ on ice. Stained cells were then washed twice and resuspended in cold buffer and analyzed with a FACScan flow cytometry (BD Biosciences, San Jose, CA, USA). More than $1 \times 10^{5}$ cells were analyzed for each sample, and the results were processed by using WinMDI 2.8 software (Scripps Research Institute, La Jolla, CA, USA).

2.9. In Vivo Efficacy Study. All experimental protocols were approved by the Institutional Animal Care and Use Committee (IACUC number: ITRI-IACUC-2012-010M, Industrial Technology Research Institute of Taiwan, HsinChu, Taiwan. SCID (CB17/Icr-Prkdcscid/CrlBltw) mice were purchased from BioLASCO Ltd. (Ilan, Taiwan). Huh-7 cells $\left(3 \times 10^{6}\right.$ cells per mice) in $100 \mu \mathrm{L}$ mix (equal volumes of PBS and Matrigel) were implanted subcutaneously (sc.) into the right flank of female SCID mice (6-8 weeks old). Tumor sizes were measured with calipers, and tumor volumes $\left(\mathrm{mm}^{3}\right)$ were calculated with the following formula: $V=L S^{2} / 2$ (where $L$ is the longest diameter and $S$ is the shortest diameter).

Huh-7 tumors were allowed to grow to $100-200 \mathrm{~mm}^{3}$. Periplocin $(5-20 \mathrm{mg} / \mathrm{kg} ; n=6)$ or a vehicle control $(n=6)$ was intraperitoneally (IP) injected into tumor bearing mice once daily for 14 days. The formula of the vehicle is $10 \% \mathrm{NMP}$ (M6762, Sigma-Aldrich, St. Louise, MO, USA), 20\% Cremophor EL (C5135, Sigma-Aldrich, St. Louise, MO, USA), and $70 \%$ Saline. Tumor volume and body weight of animals were determined twice a week. The antitumor activity of treatments was illustrated by percentage of tumor growth inhibition (TGI). TGI was calculated as [1 - (tumor volume final - tumor volume initial for treated group)/(tumor volume final - tumor volume initial for vehicle group) $] \times 100$.

2.10. Histology and Immunohistochemistry. At the end of the study, mice were sacrificed, and tumor samples were collected, fixed in formalin, and embedded in paraffin as tissue sections. Tissue sections were stained with hematoxylin and eosin ( $\mathrm{H}$ and $\mathrm{E}$ ) for general tissue morphology evaluation. The antihuman Ki67 antibody (1 : 500 dilution, IS-626, Dako, Glostrup Denmark) and antihuman cyclin-D1 antibody (1:500 dilution, IS-626, Dako, Glostrup, Denmark) were used in immunohistochemistry staining. Staining procedure was completed by using Autostainer Link 48 system (Dako,
Glostrup Denmark). Five fields of every tumor sample were randomly selected, and the percentage of the Ki67-positive and cyclin-D1-positive cells was calculated to evaluate the proliferation of tumor samples.

\section{Result}

3.1. Periplocin as the Active Ingredient in Cortex Periplocae in Inhibiting the Growth of Hepatocellular Carcinoma Cells. Cortex periplocae (CP) is a traditional medicine capable of inhibiting cancer cell growth. To identify the active ingredients in $\mathrm{CP}$ that are responsible for its activity in inhibiting the growth of hepatocellular carcinoma (HCC) cells, we isolated and purified CP. After several rounds of purification, we found a group of compounds named CP-1 to 6 as major components in the fraction that can actively inhibit tumor cell growth (Figure 1(a)).

To further identify the active ingredients in the fraction, the pure compounds in the active fraction was examined one by one, and periplocin (CP-1) was identified as the most potent compound in inhibiting tumor cell growth with $\mathrm{IC}_{50}$ at $0.027 \mu \mathrm{M}$ (Figure $1(\mathrm{~b})$ ). The cotreatment of TRAIL and periplocin or periplogenin (CP-5) strongly enhances the growth inhibiting activity of periplocin and periplogenin (Figure 1(b)). Interestingly, periplocin is less toxic to normal cells. The cell viability of PBMC was more than $80 \%$ when treated with $300 \mu \mathrm{g} / \mathrm{mL}$ periplocin (data not shown).

3.2. The Combination Treatments of Periplocin and TRAILInduced Apoptosis in TRAIL-Resistant HCC Cells. Although TRAIL is a promising anticancer drug, more and more TRAIL-resistant cancers were reported. We sought to determine if periplocin can sensitize TRAIL-resistant HCC cells to TRAIL treatment. As shown in Figure 2(a), TRAIL or periplocin alone had little effect on the viability of HCC cells, but the combination of these two drugs showed cytotoxicity to TRAIL-resistant HCC cells.

To study if the combination treatments of periplocin and TRAIL sensitize TRAIL-resistant HCC cells and induce HCC apoptosis, HA22T/VGH cells were stained with Annexin $\mathrm{V}$ and PI to characterize cells in early and late stages of apoptotic processes accordingly. As shown in Figure 2(b), periplocin treatment increased the ratio of Annexin $\mathrm{V}$ and PI positive HCC cells. Cotreatment of TRAIL and periplocin further increased the ratio of Annexin V and PI positive cells. Therefore, TRAIL and periplocin synergistically induced cell apoptosis in HCC cells.

Moreover, the accumulation of cell debris after apoptosis was demonstrated by sub-G1 population in cell cycle analysis. Consistent with our previous results, periplocin dose dependently increased sub-G1 population in HCC cells, while the addition of TRAIL further increased the sub-G1 population in HCC cells (Figure 2(c)).

\subsection{Periplocin and TRAIL Cotreatment Induces Apoptosis in} HCC Cells by Inducing DR4 Expression and Activating Pro-Apoptotic Proteins. Intracellular reactive oxygen species (ROS) are involved in apoptotic pathways. Therefore, 


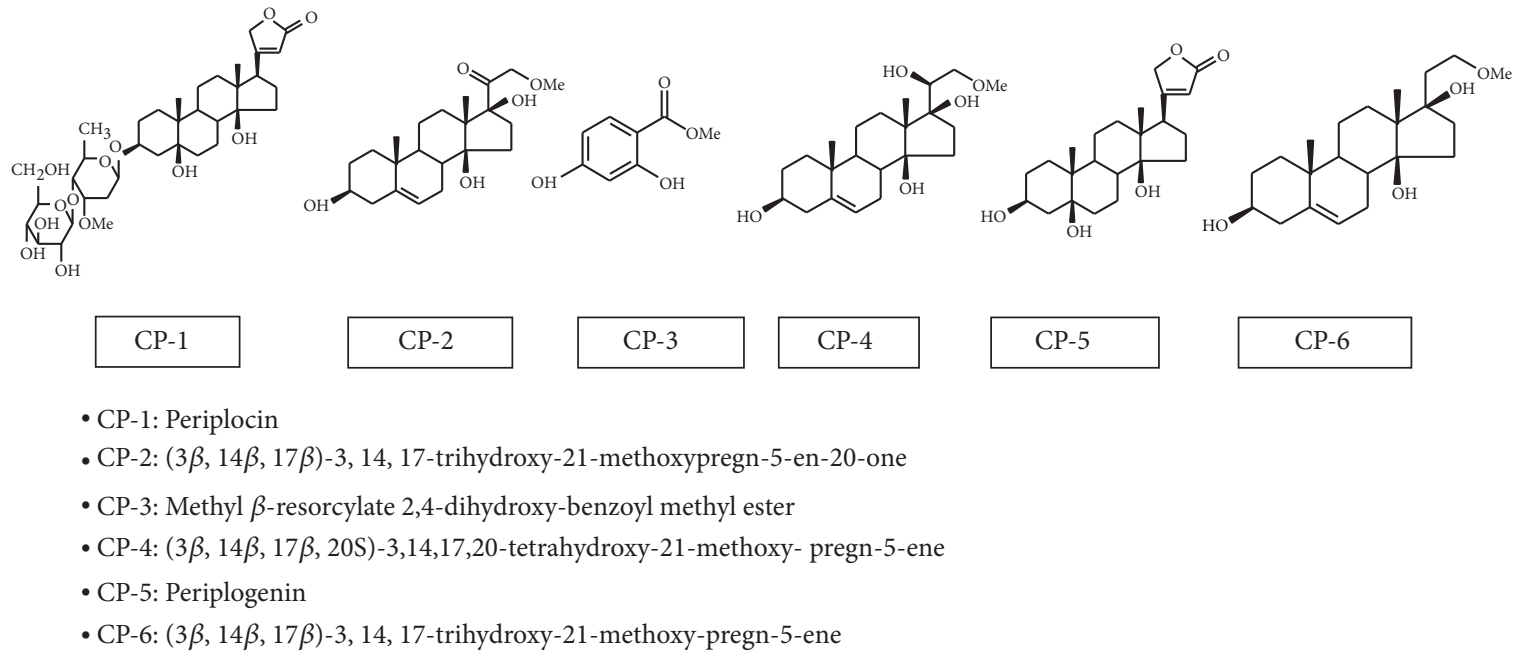

(a)
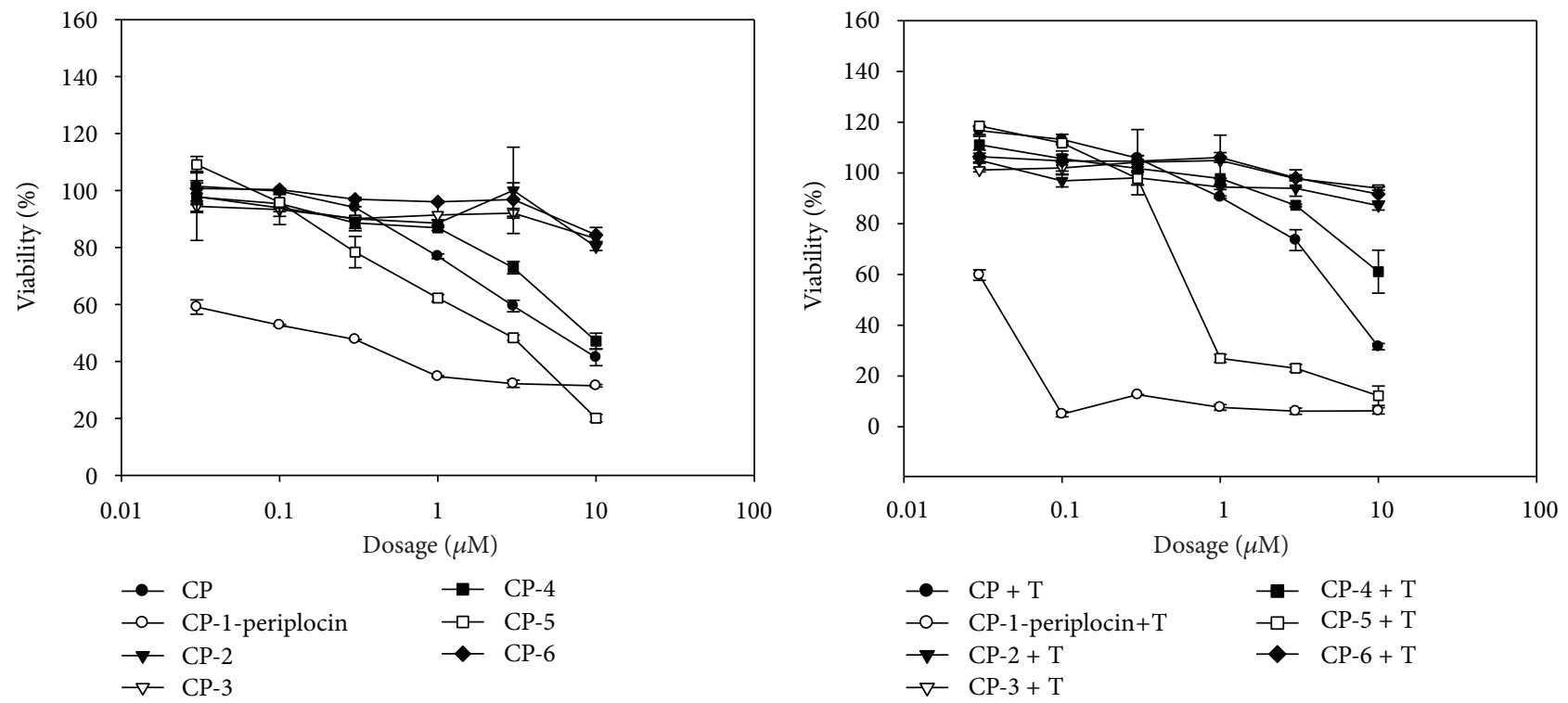

(b)

Figure 1: The effect of cortex periplocae extracts on HCC cell viability. (a) The chemical structures and names of purified cortex periplocae extracts are listed. (b) The cell viability of HA22T/VGH cells was determined by MTT assay after cells were treated with different concentrations of the indicated compound $(0.03,0.1,0.3,1,3$, and $10 \mu \mathrm{M})$ with or without TRAIL (T) for $48 \mathrm{~h}$.

the effects of periplocin and/or TRAIL treatments on the intracellular ROS level in HA22T/VGH cells were examined. Although periplocin treatment alone or together with TRAIL induced intracellular ROS accumulation in HA22T/VGH cells, NAC pretreatment did not prevent cell apoptosis induced by TRAIL and periplocin cotreatment (supplemental Figure 1 (see Supplementary Material available online at http://dx.doi.org/10.1155/2013/958025.) and data not shown)

TRAIL induces cell apoptosis through interaction with death receptors DR4 and DR5 signaling. It was reported that compounds which upregulate DR4 and DR5 in HCC cells could sensitize TRAIL-resistant HCC cells to TRAIL treatment [22]. To investigate if periplocin sensitizes
TRAIL-resistant cells through the same mechanism, the expression levels of DR4 and DR5 in HA22T/VGH cells with or without periplocin treatments were detected. Periplocin increased DR4 expression and further induced FADD expression in HA22T/VGH cells 8 hours after treatment. However, periplocin did not induce DR5 expression in HA22T/VGH cells (Figure 3(a)). The results were verified by FACS analysis (data not shown).

TRAIL binds DR4 and activates FADD. Activated FADD induces the cleavage of several proapoptotic proteins and activates them. We examined the activation of several apoptosis-related proteins, including the cleavage of BID, caspase 8 , caspase 3, and PARP in HA22T/VGH. As expected, periplocin or TRAIL treatment alone had little effects on 


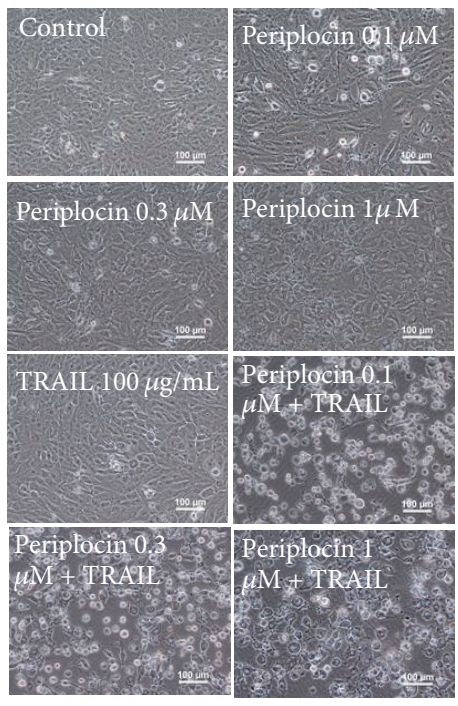

(a)

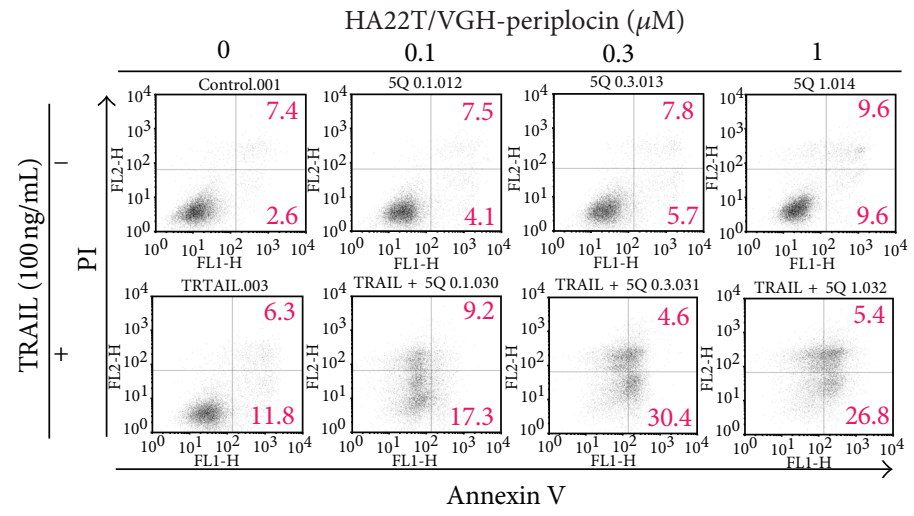

(b)

HA22T/VGH-periplocin $(\mu \mathrm{M})$

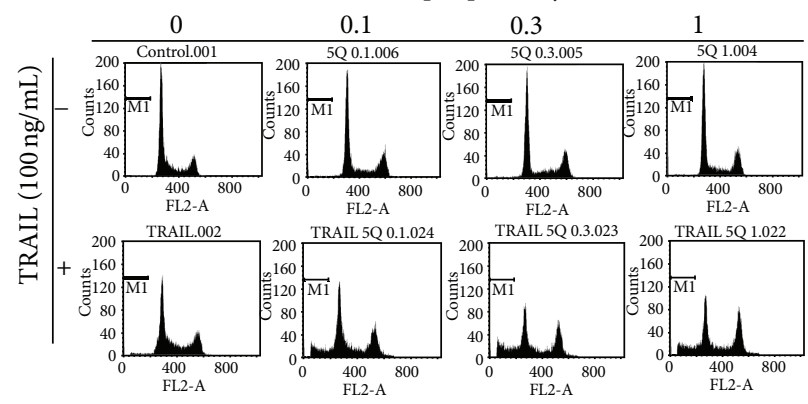

Huh7-periplocin (um)

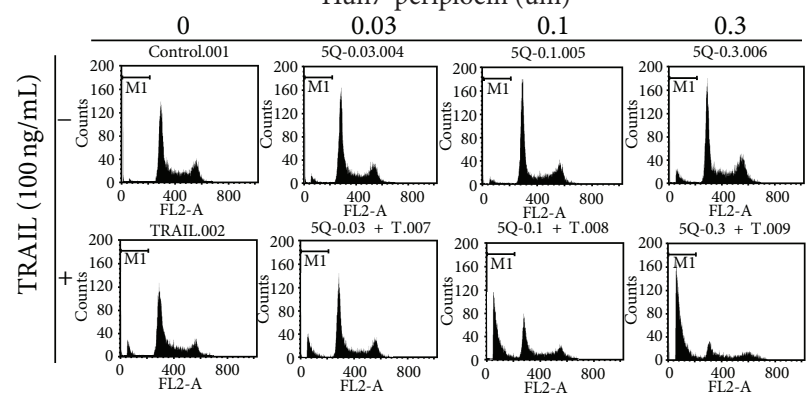

(c)

FIGURE 2: Periplocin and TRAIL treatments regulated cell apoptosis in HA22T/VGH cells. (a) HA22T/VGH cells were treated with periplocin alone or together with TRAIL for $24 \mathrm{~h}$, and the morphology of cells was observed under microscope. (b) Cell apoptosis in HA22T/VGH cells regulated by periplocin and/or TRAIL was determined by FACS with Annexin-V-Fluorescein ( $x$-axis) and PI ( $y$-axis) double-staining system. (c) PI staining for both HA22T/VGH and Huh-7 cells after periplocin and/or TRAIL treatments. Apoptotic cells were quantified by FACS analysis (sub-G1 group).

the cleavage of BID, caspase 8 , caspase 3, and PARP. The combination of periplocin and TRAIL treatments strongly increase the cleavage of all these apoptosis-related proteins (Figure 3(b)). Similar results were observed in Huh7, another HCC cell line (Figure 3(c)).

To further confirm the importance of caspase activation in periplocin-regulated cell apoptosis with or without TRAIL treatment, caspase inhibitors were added to HA22T/VGH cells prior to periplocin and/or TRAIL treatments. Inhibitors against caspase 3 , caspase 8 , and caspase 9 partially rescued cell survival repressed by periplocin and/or TRAIL treatments, and pan inhibitor against all three caspases completely blocked cell death induced by periplocin and/or TRAIL treatments (Figure 3(d)).

3.4. Periplocin and TRAIL Cotreatment Induces Apoptosis in HA22T/VGH Cells by Inhibiting IAPs. Another reported mechanism for sensitizing TRAIL-resistant cell lines to 


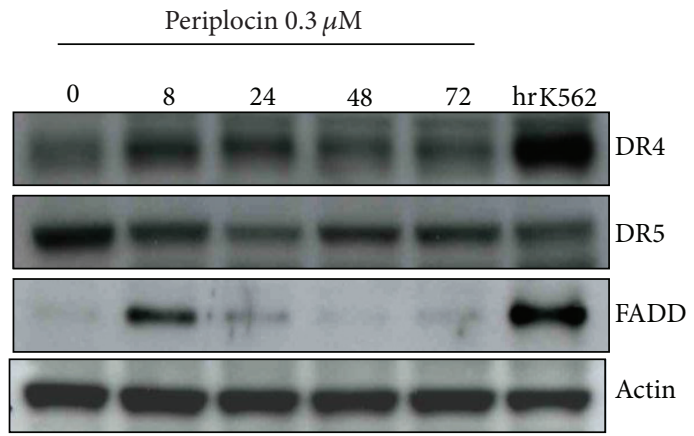

(a)

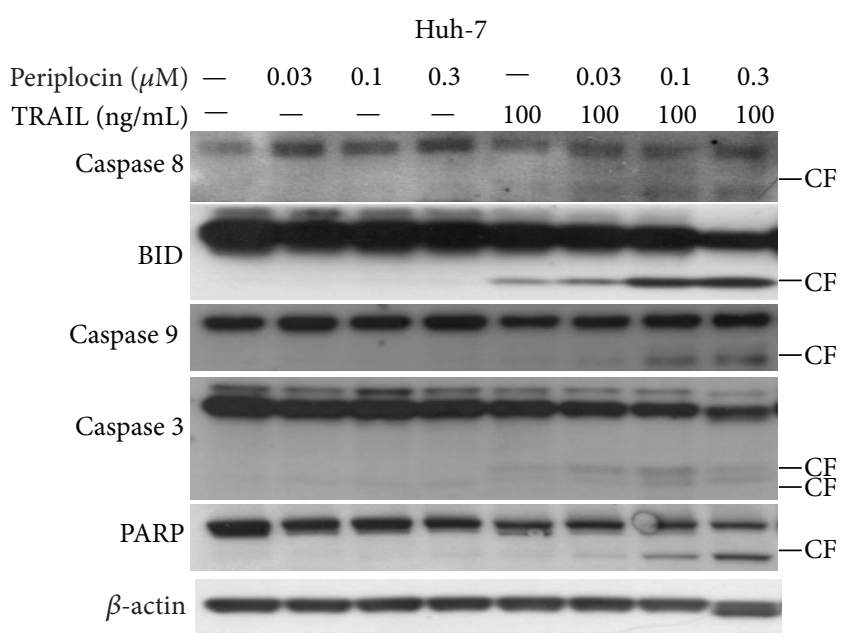

(c)

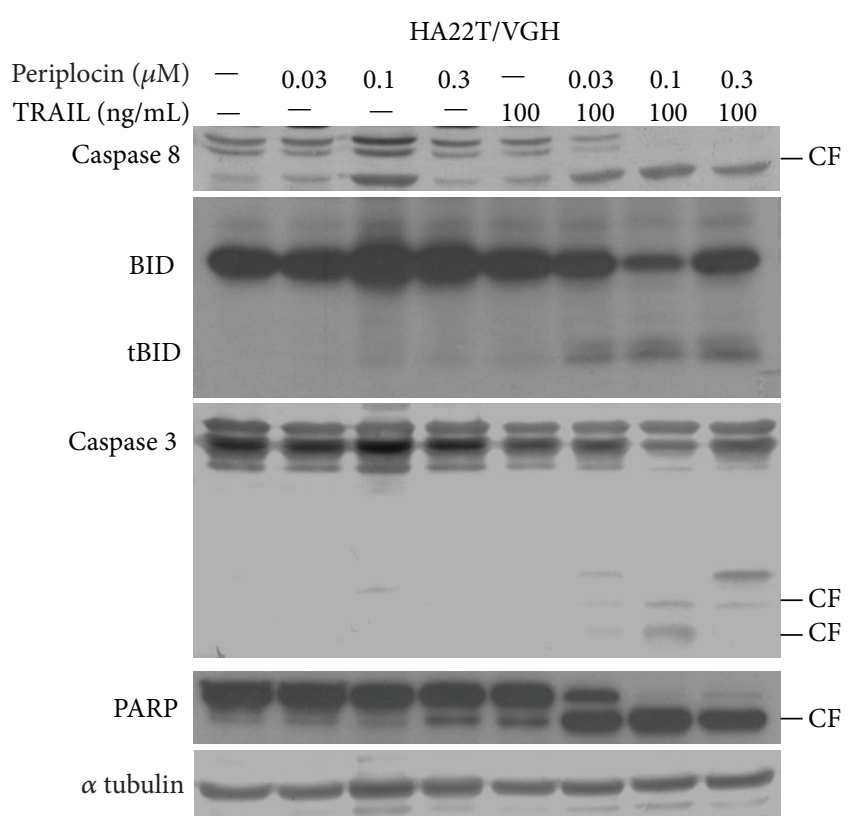

(b)

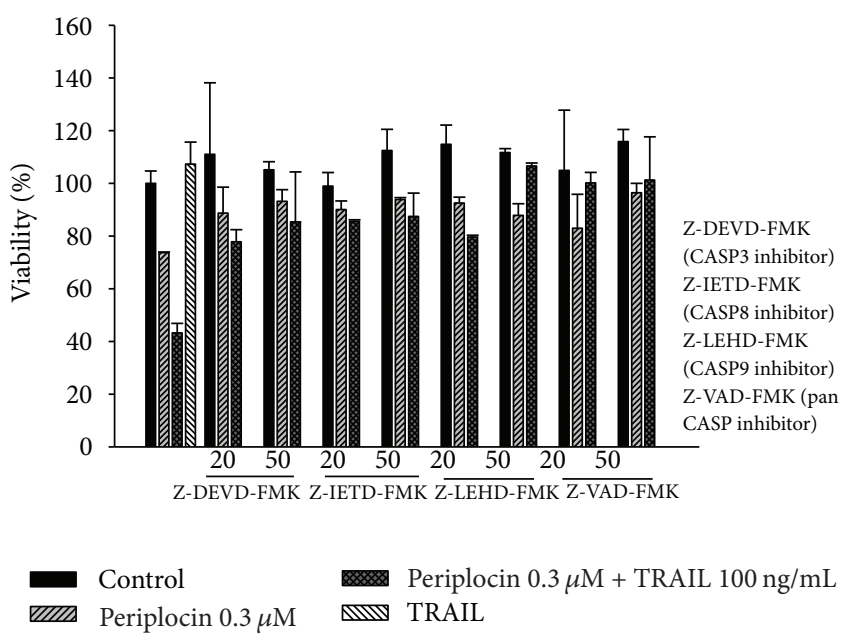

(d)

FIGURE 3: Treatments of periplocin and/or TRAIL activate DR4, FADD, and proapoptotic proteins in HCC cells. (a) The effect of periplocin treatment on the expression of DR4, DR5, and FADD was analyzed by western blot. (b) HA22T/VGH cells were treated with different doses of the indicated compounds for $24 \mathrm{~h}$. Expressions of both proforms and cleaved forms of caspase- 8 , caspase 3 , PARP, and BID were analyzed by western blotting. (c) Huh-7 cells were treated with different concentrations of the indicated compounds for $4 \mathrm{~h}$ or $24 \mathrm{~h}$. The expression of caspase-8, caspase- 9 was analyzed after indicated compounds treatment for $4 \mathrm{~h}$, and the expression of caspase 3, PARP, BID was analyzed after the treatment of indicated compounds for $24 \mathrm{~h}$. (d) HA22T/VGH cells were pretreated with $20 \mu \mathrm{M}$ or $50 \mu \mathrm{M}$ inhibitors against caspase-3 (Z-DEVD-FMK), caspasae-8 (Z-IETD-FMK), caspase-9 (Z-LEHD-FMK), and general caspase inhibitor (Z-VAD-FMK) for 3 hours prior to periplocin and/or TRAIL treatment. Cell viability was examined by MTT assay.

TRAIL treatment is through regulating proteins involved in apoptotic pathways [23]. Since the cleavage of caspases can be induced either through intrinsic (mitochondrial mediated) or extrinsic (death receptor mediated) apoptotic pathways, the role of mitochondrial-mediated caspase cleavage was first investigated in HA22T/VGH with periplocin and TRAIL treatments. The expression levels of $\mathrm{Bcl}-2$ protein family and several mitochondria dependent apoptotic regulators, including Bax, Bad, Mcl-1, and apaf-1, were detected before and after periplocin and/or TRAIL treatments. However, the treatments of periplocin and/or TRAIL did not affect the expression of apoptotic regulators (Figure 4(a) and supplemental Figure 2A). Nevertheless, the combination treatment of periplocin and TRAIL activated caspase 9 in two different HCC cell lines (Figures 3(c) and 4(a) and supplemental Figure 2 ). Since the combination treatment of periplocin and TRAIL activated several caspases, we examined the effect of periplocin and TRAIL on members of inhibitors 


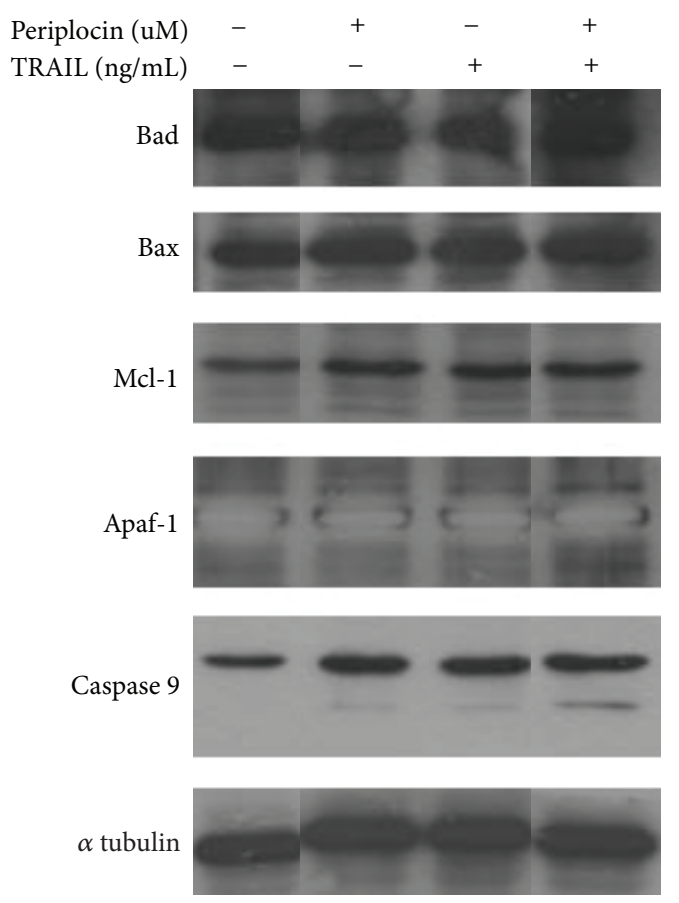

(a)

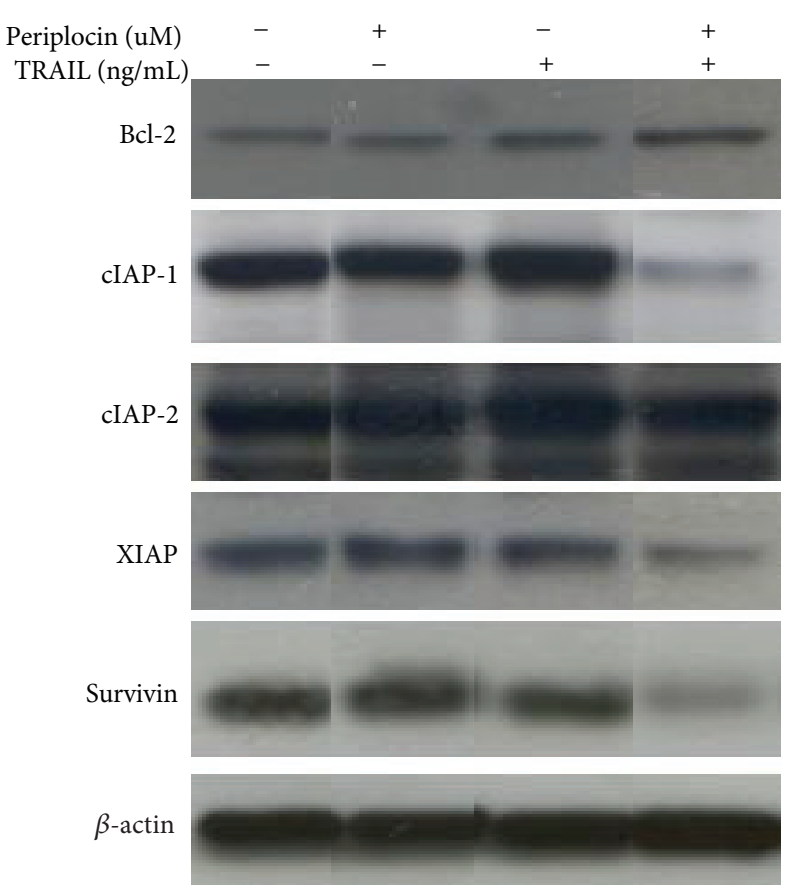

(b)

FIGURE 4: Cotreatment of periplocin and TRAIL activated IAP. (a) The expression levels of Bax, Bad, Mcl-1, apaf-1, and caspase 9 in HA22T/VGH in response to $1 \mu \mathrm{M}$ periplocin and/or $100 \mathrm{ng} / \mathrm{mL}$ TRAIL treatment were examined by western blot. (b) The expression levels of $\mathrm{Bcl}-2$, cIAP-1, cIAP-2, XIAP, and survivin in HA22T/VGH in response to $1 \mu \mathrm{M}$ periplocin and/or $100 \mathrm{ng} / \mathrm{mL}$ TRAIL treatment were examined by western blot. The original blots are shown in supplemental Figure 2 .

of apoptosis (IAP) family. The expression of several IAP family members, including CIAP-1, XIAP, and survivin, was repressed by the combination treatment of periplocin and TRAIL in HA22T/VGH cells (Figure 4(b) and supplemental Figure 2 ).

3.5. Periplocin Represses Tumor Formation In Vivo. In addition to in vitro mechanistic studies, we also verified the potency of TRAIL and periplocin on repressing tumor growth in vivo. Since TRAIL was expressed in NK cells in mice, we treated tumor-bearing mice with only periplocin in this in vivo study $[24,25]$. To test the antitumor activity of periplocin in vivo, HCC cells were subcutaneously injected into SCID mice, and periplocin was intraperitoneally (IP) injected daily two weeks after the initial injection of tumor cells. Since the mice were well-tolerant to $5 \mathrm{mg} / \mathrm{kg}$ periplocin after 2 weeks of treatment, the dose of periplocin were raised to $20 \mathrm{mg} / \mathrm{kg}$ daily throughout the study. Periplocin was able to inhibit HCC growth in xenograft model. Tumor growth inhibition (TGI, \%) of periplocin treatment was $51 \pm 11 \%$ after 24 days treatment, (Figure 5(a)). When treated with periplocin, the mice body weight was slightly decreased at the dose of $20 \mathrm{mg} / \mathrm{kg}$ when compared to the vehicle group. However, the body weight kept at around 90 percent of control group, and no further body weight loss was observed (Figure 5(b)). These data showed that periplocin strongly inhibited tumor growth of Huh-7 tumors without obvious side effects.
In Ki67 immunochemistry analysis, periplocin could inhibit the Ki67 expression in tumor samples (Figure 5(c)). After selecting five random fields and calculating the Ki67 positive cells of every tumor sample, the percentage of Ki67-positive cells in vehicle group was $57.3 \pm 0.67 \%$. The percentage of Ki67-positive cells in periplocin-treated group was $22.78 \pm 10.09 \%$. The result showed that periplocin could significantly inhibit the Ki67 expression in Huh-7 tumors, which suggested that periplocin inhibited tumor growth in vivo.

To further verify the periplocin-inhibited tumor growth in vivo, we also examined the expression of cyclin-D1 in Huh-7 tumors. In cyclin-D1 immunochemistry analysis, the percentage of cyclin-D1-positive cells in vehicle group was $76.87 \pm 2.93 \%$. The percentage of cyclin-D1-positive cells in periplocin-treated group was $58.85 \pm 5.05 \%$. The result showed that periplocin could inhibit the cyclin-D1 expression in tumor samples (Figure 5(c)). To verify the role of periplocin in cyclin-D1 expression, Huh-7 cells were seeded on Millipore Millicell EZ slide $(2 \times 10 \mathrm{e} 4 /$ well $)$ for $24 \mathrm{~h}$ and subsequently treated with different concentrations of periplocin for $24 \mathrm{~h}$. As expected, periplocin dosedependently repressed cyclin-D1 expression in Huh-7 cells (Figure 5(d)).

\section{Discussion}

Periplocin is a cardiac glycoside structurally similar to digoxin. The pharmacological function of periplocin is also 


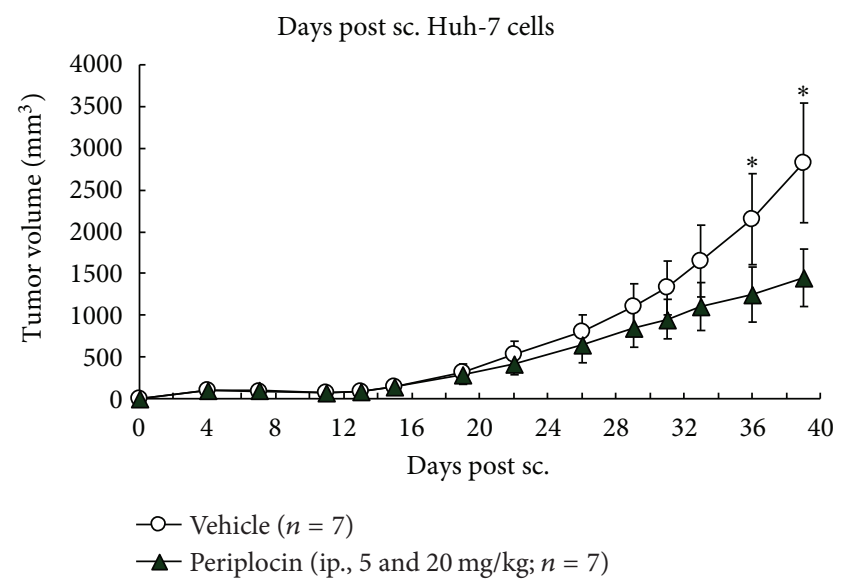

(a)
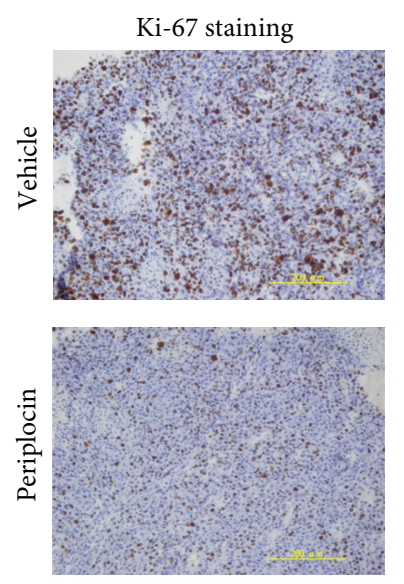

(c)

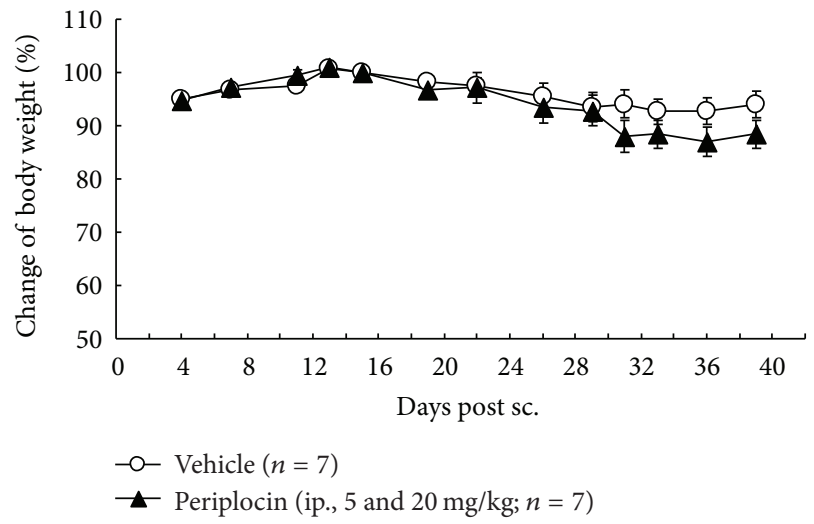

(b)

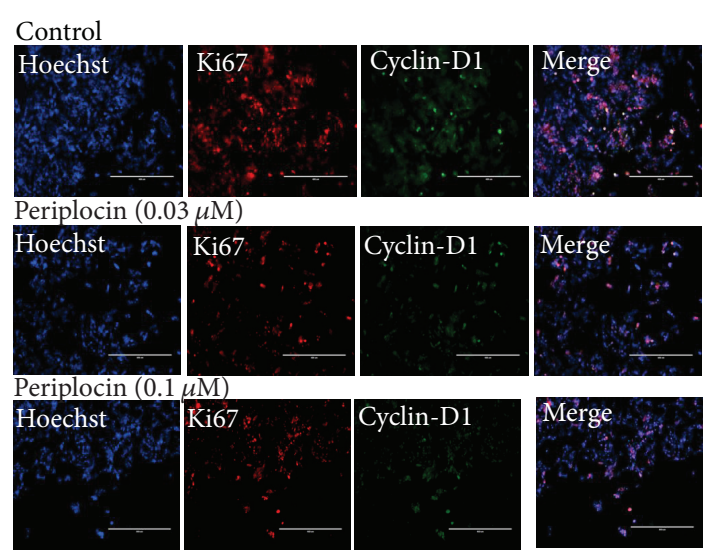

(d)

FIGURE 5: Periplocin treatments affected tumor growth in xenograft in vivo model. HCC cells were subcutaneously injected into SCID mice at $3 \times 10^{6}$ cells. Periplocin was IP injected into SCID mice daily two weeks after initial tumor injection $(5 \mathrm{mg} / \mathrm{kg}$ on day 15 to day 29 and $20 \mathrm{mg} / \mathrm{kg}$ on day 29 to day 35). (a) Tumor size was measured every $2-4$ days throughout the study $\left({ }^{*} P<0.05\right)$. (b) Body weight of control and treated mice were measured every 2-4 days throughout the study. (c) After the mice were sacrificed, tissue sections were prepared and stained with anti-Ki67 and anticyclin-D1 antibodies to evaluate cell proliferation. (d) Huh-7 cells were fixed, and immunofluorescent stained by Hoechst 33258 (blue staining), Ki67 (red staining), and cyclin-D1 (green staining).

similar to digoxin and has been used to treat heart diseases. Nevertheless, digoxin was shown to block cancer growth through inhibiting HIF- $1 \alpha$ signaling pathway in cancer cells [26]. Indeed, Digitalis was used as treatment for breast cancer patients and reduced cancer recurrence rate $[27,28]$. Compounds structurally similar to digoxin also possess antitumor activity [29]. Several studies suggest that cardiac glycoside induces cell apoptosis. Digoxin induces apoptosis through activating Cdk5 [30]. In addition, Digitalis was reported to induce mitochondria-dependent apoptotic pathways in guinea-pig cardiomyocytes [31].

TRAIL induces cell apoptosis via DR4 and DR5, activates Fas-associated death domain (FADD) and caspase 8 , and signals through both mitochondria-dependent and -independent pathways [32]. There are three antagonistic decoy receptors DcR1, DcR2, and osteoprotegerin that interact with TRAIL but cannot transmit apoptotic signal. Therefore, cancer cells could gain TRAIL-resistance by overexpressing DcR1, DcR2, or osteoprotegerin [33-35]. In addition, defects in adaptor protein FADD or caspase 8 can also lead to TRAIL resistance $[36,37]$. In this study, we demonstrated that periplocin induced DR4 and FADD expression in TRAIL-resistant HCC cells. The combination treatment of TRAIL and periplocin activated caspase 8 , the key caspase for both mitochondria-dependent andindependent apoptotic signaling pathways, in TRAIL resistant HCC cells.

The combination treatment of periplocin and TRAIL induced HCC cell apoptosis through activating IAP. IAPs are members of a protein family that regulate apoptosis. Currently there are 8 known members in this family, and $\mathrm{X}$-linked inhibitor of apoptosis protein (XIAP) is the best characterized member in the family. XIAP blocks apoptosis by binding and inactivating caspase 3, 7, and 9 [38]. Other IAP, including c-IAPl, c-IAP2, and survivin, have been shown to bind to caspase 3 and 7 [39]. Indeed, we observed the 


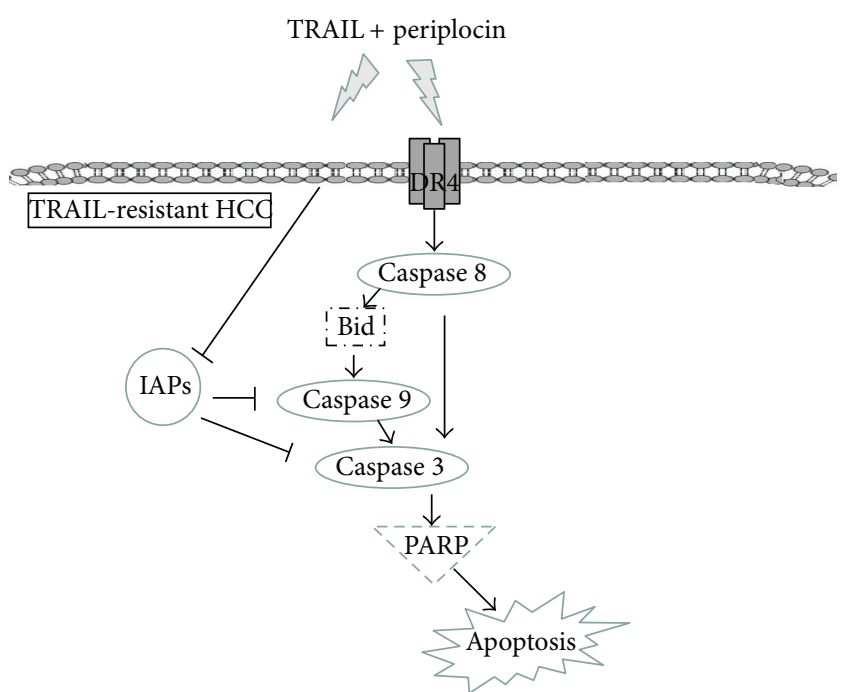

FIGURE 6: Mechanisms of resensitizing TRAIL-resistant HCC cells by periplocin. Periplocin can resensitize TRAIL resistant HCC by inducing the expression of DR4. Therefore, the combination treatments of TRAIL and periplocin can induce cell apoptosis through direct activation of caspase signaling and indirect inhibition of IAPs.

repression of XIAP, c-IAP1, and survivin, and the deactivation of caspase 3 and 9 by TRAIL and periplocin treatments in this study.

The expression of TRAIL by NK cells was demonstrated both in vitro and in vivo $[24,25,40,41]$. We took advantage of that and designed an experiment that combined endogenous TRAIL and exogenous periplocin treatment. The injected periplocin was able to repress HCC tumor growth in vivo.

While purifying cortex periplocae, we identified six compounds with potential antitumor activity (Figure 1). Among these compounds, periplocin (CP-1) and periplogenin (CP5 ) are the two compounds with cytotoxicity against cancer cells, and periplocin is more potent than periplogenin. Structurally, periplocin is different from periplogenin by only one disaccharide residue. It was reported that saccharide residues are involved in the recognition of plant root surfaces by zoospores [42]. Therefore, it is not surprising that saccharide residues can affect protein interactions. However, detailed mechanisms of how periplocin interacts with cell surface molecules and how does the disaccharide residue regulate cell apoptosis require further investigation.

In traditional herb medicine, patients are usually treated with multiple raw materials. Sometimes it is hard to purify one single active ingredient since multiple components are required to achieve therapeutic goals. In this study, we purified an active ingredient, periplocin, with cytotoxicity against HCC cells from cortex periplocae. Interestingly, maximum cytotoxicity against TRAIL-resistant HCC was achieved by combining the treatment of periplocin and TRAIL. The idea of the combination treatment is consistent with the concept of traditional medicine. Provided the mechanistic studies, the combination treatments of active ingredients from herb medicine and chemical synthesized compounds or protein drugs could be potential treatment options for drug-resistant cancers.

\section{Conclusion}

In this study, we demonstrated that periplocin could sensitize TRAIL-resistant HCC to TRAIL treatment, and the combination treatment of TRAIL and periplocin can induce apoptosis in TRAIL-resistant HCC. Furthermore, we showed that periplocin sensitized TRAIL-resistant HCC cell lines to TRAIL through the following two mechanisms. First, periplocin induced the expression of DR4 and FADD to activate proapoptotic signaling pathways. Second, the cotreatment of TRAIL and periplocin suppressed several IAP, which also led to the activation of proapoptotic signaling pathways. Our working model is shown in Figure 6. Further studies are required to apply periplocin clinically.

\section{Conflict of Interests}

The authors claim no conflict of interests.

\section{References}

[1] A. Jemal, R. Siegel, J. Xu, and E. Ward, "Cancer statistics, 2010," CA: A Cancer Journal for Clinicians, vol. 60, pp. 277-300, 2010.

[2] J. M. Llovet, S. Ricci, V. Mazzaferro et al., "Sorafenib in advanced hepatocellular carcinoma," The New England Journal of Medicine, vol. 359, no. 4, pp. 378-390, 2008.

[3] M. Numata, A. Yamamoto, A. Moribayashi, and H. Yamada, "Antitumor components isolated from the Chinese herbal medicine Coix lachryma-jobi," Planta Medica, vol. 60, no. 4, pp. 356-359, 1994.

[4] M. Su, H. Y. Chung, and Y. Li, "6-O-Angeloylenolin induced cell-cycle arrest and apoptosis in human nasopharyngeal cancer cells," Chemico-Biological Interactions, vol. 189, pp. 167-176, 2011.

[5] H. Ohba, S. Moriwaki, R. Bakalova, S. Yasuda, and N. Yamasaki, "Plant-derived abrin-a induces apoptosis in cultured leukemic cell lines by different mechanisms," Toxicology and Applied Pharmacology, vol. 195, pp. 182-193, 2004.

[6] R. V. Priyadarsini, R. S. Murugan, P. Sripriya, D. Karunagaran, and S. Nagini, "The neem limonoids azadirachtin and nimbolide induce cell cycle arrest and mitochondria-mediated apoptosis in human cervical cancer (HeLa) cells," Free Radical Research, vol. 44, no. 6, pp. 624-634, 2010.

[7] L. Zhao, B. Shan, Y. Du, M. Wang, L. Liu, and F. Z. Ren, "Periplocin from Cortex periplocae inhibits cell growth and down-regulates survivin and c-myc expression in colon cancer in vitro and in vivo via $\beta$-catenin/TCF signaling," Oncology Reports, vol. 24, no. 2, pp. 375-383, 2010.

[8] Z. J. Lu, Y. Zhou, Q. Song et al., "Periplocin inhibits growth of lung cancer in vitro and in vivo by blocking AKT/ERK signaling pathways," Cellular Physiology and Biochemistry, vol. 26, pp. 609-618, 2010.

[9] S. R. Wiley, K. Schooley, P. J. Smolak et al., "Identification and characterization of a new member of the TNF family that induces apoptosis," Immunity, vol. 3, no. 6, pp. 673-682, 1995. 
[10] G. Pan, K. O’Rourke, A. M. Chinnaiyan et al., "The receptor for the cytotoxic ligand TRAIL," Science, vol. 276, no. 5309, pp. 111-113, 1997.

[11] P. M. Chaudhary, M. Eby, A. Jasmin, A. Bookwalter, J. M. Urray, and L. Hood, "Death receptor 5, a new member of the TNFR family, and DR4 induce FADD- dependent apoptosis and activate the NF- $\kappa$ B pathway," Immunity, vol. 7, no. 6, pp. 821-830, 1997.

[12] M. J. Smyth, K. Takeda, Y. Hayakawa, J. J. Peschon, M. R. van den Brink, and H. Yagita, "Nature's TRAIL—on a path to cancer immunotherapy," Immunity, vol. 18, pp. 1-6, 2003.

[13] E. Cretney, A. Shanker, H. Yagita, M. J. Smyth, and T. J. Sayers, "TNF-related apoptosis-inducing ligand as a therapeutic agent in autoimmunity and cancer," Immunology \& Cell Biology, vol. 84, pp. 87-98, 2006.

[14] C. M. M. Van Geelen, E. G. E. De Vries, and S. De Jong, "Lessons from TRAIL-resistance mechanisms in colorectal cancer cells: paving the road to patient-tailored therapy," Drug Resistance Updates, vol. 7, no. 6, pp. 345-358, 2004.

[15] Y. Zhang and B. Zhang, "TRAIL resistance of breast cancer cells is associated with constitutive endocytosis of death receptors 4 and 5," Molecular Cancer Research, vol. 6, pp. 1861-1871, 2008.

[16] K. Chen, Y. Tu, Y. Zhang, H. C. Blair, L. Zhang, and C. Wu, "PINCH-1 regulates the ERK-Bim pathway and contributes to apoptosis resistance in cancer cells," The Journal of Biological Chemistry, vol. 283, pp. 2508-2517, 2008.

[17] S. Khanbolooki, S. T. Nawrocki, T. Arumugam et al., "Nuclear factor- $\kappa \mathrm{B}$ maintains TRAIL resistance in human pancreatic cancer cells," Molecular Cancer Therapeutics, vol. 5, no. 9, pp. 2251-2260, 2006.

[18] L. Zhang, H. Zhu, J. J. Davis et al., "Lack of p38 MAP kinase activation in TRAIL-resistant cells is not related to the resistance to TRAIL-mediated cell death," Cancer Biology and Therapy, vol. 3, no. 3, pp. 296-301, 2004.

[19] R. R. Rosato, J. A. Almenara, S. Coe, and S. Grant, “The multikinase inhibitor sorafenib potentiates TRAIL lethality in human leukemia cells in association with Mcl-1 and cFLIPL downregulation," Cancer Research, vol. 67, no. 19, pp. 9490-9500, 2007.

[20] J. F. Yang, J. G. Cao, L. Tian, and F. Liu, “5, 7-Dimethoxyflavone sensitizes TRAIL-induced apoptosis through DR5 upregulation in hepatocellular carcinoma cells," Cancer Chemotherapy and Pharmacology, vol. 69, pp. 195-206, 2012.

[21] C. H. Ko, S. C. Shen, and Y. C. Chen, "Hydroxylation at C4' or C6 is essential for apoptosis-inducing activity of flavanone through activation of the caspase- 3 cascade and production of reactive oxygen species," Free Radical Biology and Medicine, vol. 36, no. 7, pp. 897-910, 2004.

[22] O. Pellerito, G. Calvaruso, P. Portanova et al., "The synthetic cannabinoid WIN 55,212-2 sensitizes hepatocellular carcinoma cells to tumor necrosis factor-related apoptosis-inducing ligand (TRAIL)-induced apoptosis by activating p8/CCAAT/enhancer binding protein homologous protein $(\mathrm{CHOP}) /$ death receptor 5 (DR5) axis," Molecular Pharmacology, vol. 77, no. 5, pp. 854-863, 2010.

[23] W. Ding, T. Cai, H. Zhu et al., "Synergistic antitumor effect of TRAIL in combination with sunitinib in vitro and in vivo," Cancer Letters, vol. 293, pp. 158-166, 2010.

[24] Y. Hayakawa, V. Screpanti, H. Yagita et al., "NK cell TRAIL eliminates immature dendritic cells in vivo and limits dendritic cell vaccination efficacy," Journal of Immunology, vol. 172, no. 1, pp. 123-129, 2004.
[25] M. J. Smyth, E. Cretney, K. Takeda et al., "Tumor necrosis factor-related apoptosis-inducing ligand (TRAIL) contributes to interferon $\gamma$-dependent natural killer cell protection from tumor metastasis," Journal of Experimental Medicine, vol. 193, no. 6, pp. 661-670, 2001.

[26] H. Zhang, D. Z. Qian, Y. S. Tan et al., "Digoxin and other cardiac glycosides inhibit HIF- $1 \alpha$ synthesis and block tumor growth," Proceedings of the National Academy of Sciences of the United States of America, vol. 105, no. 50, pp. 19579-19586, 2008.

[27] B. Stenkvist, "Is digitalis a therapy for breast carcinoma?" Oncology Reports, vol. 6, pp. 493-496, 1999.

[28] B. Stenkvist, E. Pengtsson, B. Dahlqvist, O. Eriksson, T. Jarkrans, and B. Nordin, "Cardiac glycosides and breast cancer, revisited," The New England Journal of Medicine, vol. 306, no. 8, article 484, 1982.

[29] F. Qi, Y. Inagaki, B. Gao et al., "Bufalin and cinobufagin induce apoptosis of human hepatocellular carcinoma cells via Fas- and mitochondria-mediated pathways," Cancer Science, vol. 102, no. 5, pp. 951-958, 2011.

[30] H. Lin, J. L. Juang, and P. S. Wang, "Involvement of Cdk5/p25 in digoxin-triggered prostate-cancer cell apoptosis," Journal of Biological Chemistry, vol. 279, no. 28, pp. 29302-29307, 2004.

[31] M. Ramirez-Ortega, G. Zarco, V. Maldonado et al., "Is digitalis compound-induced cardiotoxicity, mediated through guineapig cardiomyocytes apoptosis?" European Journal of Pharmacology, vol. 566, no. 1-3, pp. 34-42, 2007.

[32] A. Suliman, A. Lam, R. Datta, and R. K. Srivastava, "Intracellular mechanisms of TRAIL: apoptosis through mitochondrialdependent and -independent pathways," Oncogene, vol. 20, no. 17, pp. 2122-2133, 2001.

[33] M. A. Degli-Esposti, P. J. Smolak, H. Walczak et al., "Cloning and characterization of TRAIL-R3, a novel member of the emerging TRAIL receptor family," Journal of Experimental Medicine, vol. 186, no. 7, pp. 1165-1170, 1997.

[34] M. A. Degli-Esposti, W. C. Dougall, P. J. Smolak, J. Y. Waugh, C. A. Smith, and R. G. Goodwin, "The novel receptor TRAIL$\mathrm{R} 4$ induces NF- $\kappa \mathrm{B}$ and protects against TRAIL- mediated apoptosis, yet retains an incomplete death domain," Immunity, vol. 7, no. 6, pp. 813-820, 1997.

[35] J. G. Emery, P. McDonnell, M. B. Burke et al., "Osteoprotegerin is a receptor for the cytotoxic ligand TRAIL," Journal of Biological Chemistry, vol. 273, no. 23, pp. 14363-14367, 1998.

[36] A. Eggert, M. A. Grotzer, T. J. Zuzak et al., "Resistance to tumor necrosis factor-related apoptosis-inducing ligand (TRAIL)induced Apoptosis in neuroblastoma cells correlates with a loss of caspase-8 expression," Cancer Research, vol. 61, no. 4, pp. 1314-1319, 2001.

[37] P. T. Daniel, T. Wieder, I. Sturm, and K. Schulze-Osthoff, "The kiss of death: promises and failures of death receptors and ligands in cancer therapy," Leukemia, vol. 15, no. 7, pp. 1022-1032, 2001.

[38] Q. L. Deveraux and J. C. Reed, "IAP family proteins-suppressors of apoptosis," Genes \& Development, vol. 13, pp. 239-252, 1999.

[39] I. Tamm, Y. Wang, E. Sausville et al., "IAP-family protein Survivin inhibits caspase activity and apoptosis induced by Fas (CD95), bax, caspases, and anticancer drugs," Cancer Research, vol. 58, no. 23, pp. 5315-5320, 1998.

[40] S. R. Wiley, K. Schooley, P. J. Smolak et al., "Identification and characterization of a new member of the TNF family that induces apoptosis," Immunity, vol. 3, no. 6, pp. 673-682, 1995. 
[41] P. Mirandola, C. Ponti, G. Gobbi et al., "Activated human NK and $\mathrm{CD}^{+} \mathrm{T}$ cells express both TNF-related apoptosisinducing ligand (TRAIL) and TRAIL receptors but are resistant to TRAIL-mediated cytotoxicity," Blood, vol. 104, no. 8, pp. 2418-2424, 2004.

[42] D. Longman and J. A. Callow, "Specific saccharide residues are involved in the recognition of plant root surfaces by zoospores of Pythium aphanidermatum," Physiological and Molecular Plant Pathology, vol. 30, no. 1, pp. 139-150, 1987. 


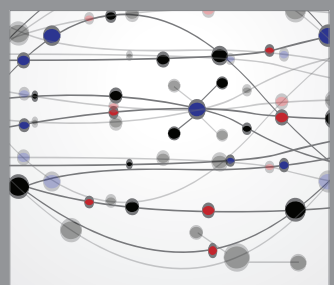

The Scientific World Journal
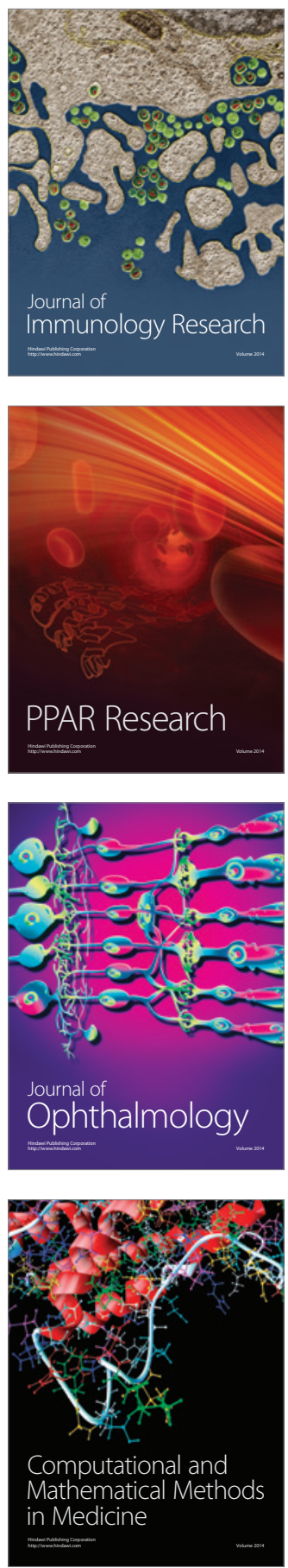

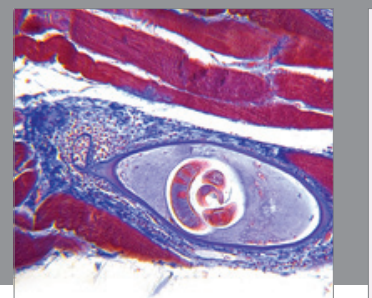

Gastroenterology

Research and Practice
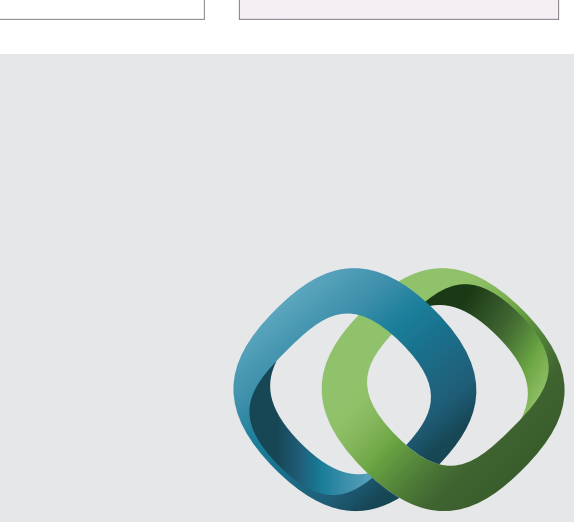

\section{Hindawi}

Submit your manuscripts at

http://www.hindawi.com
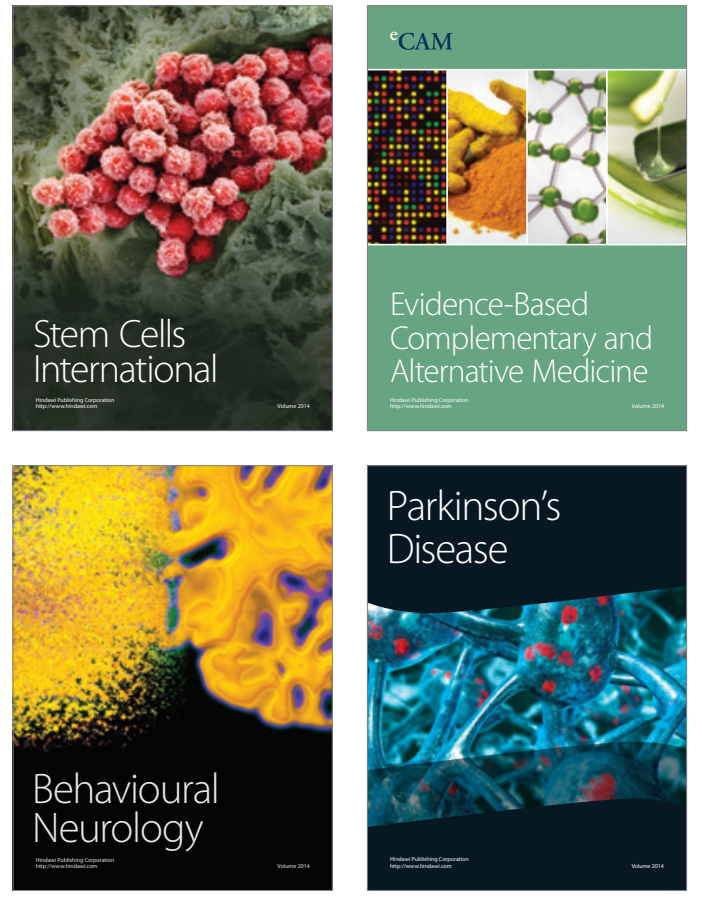
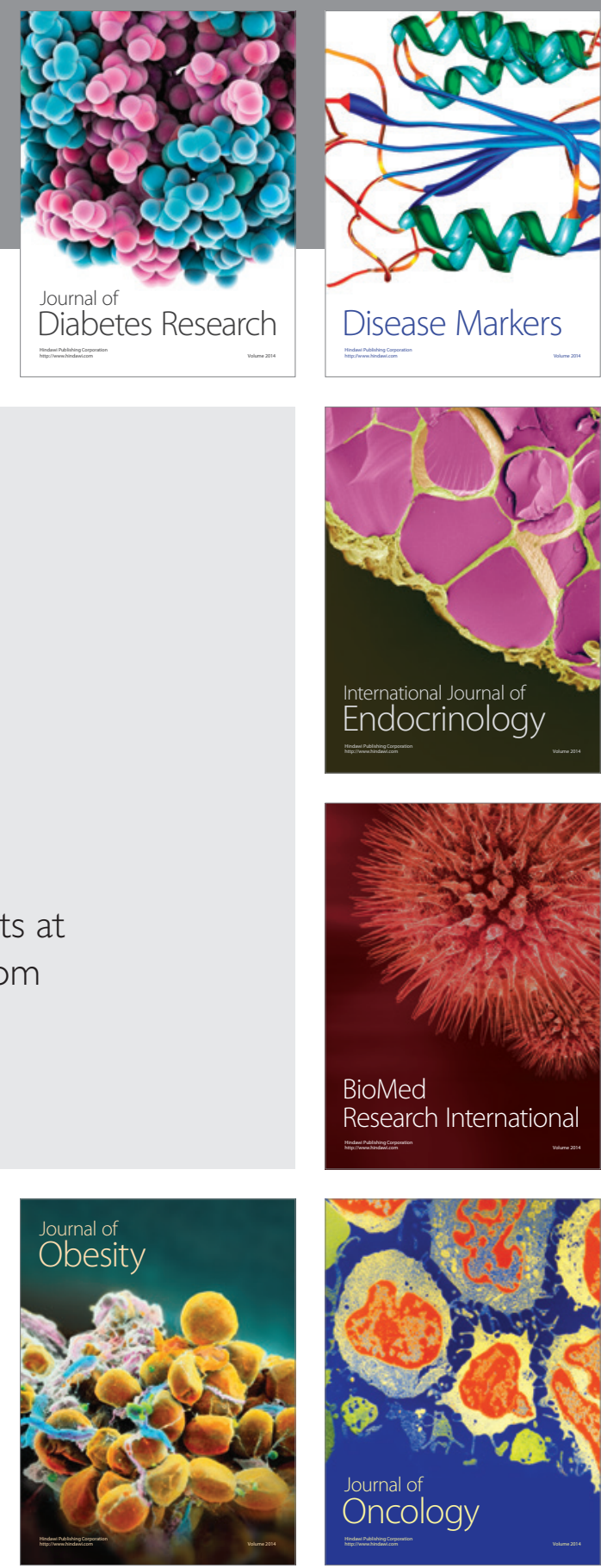

Disease Markers
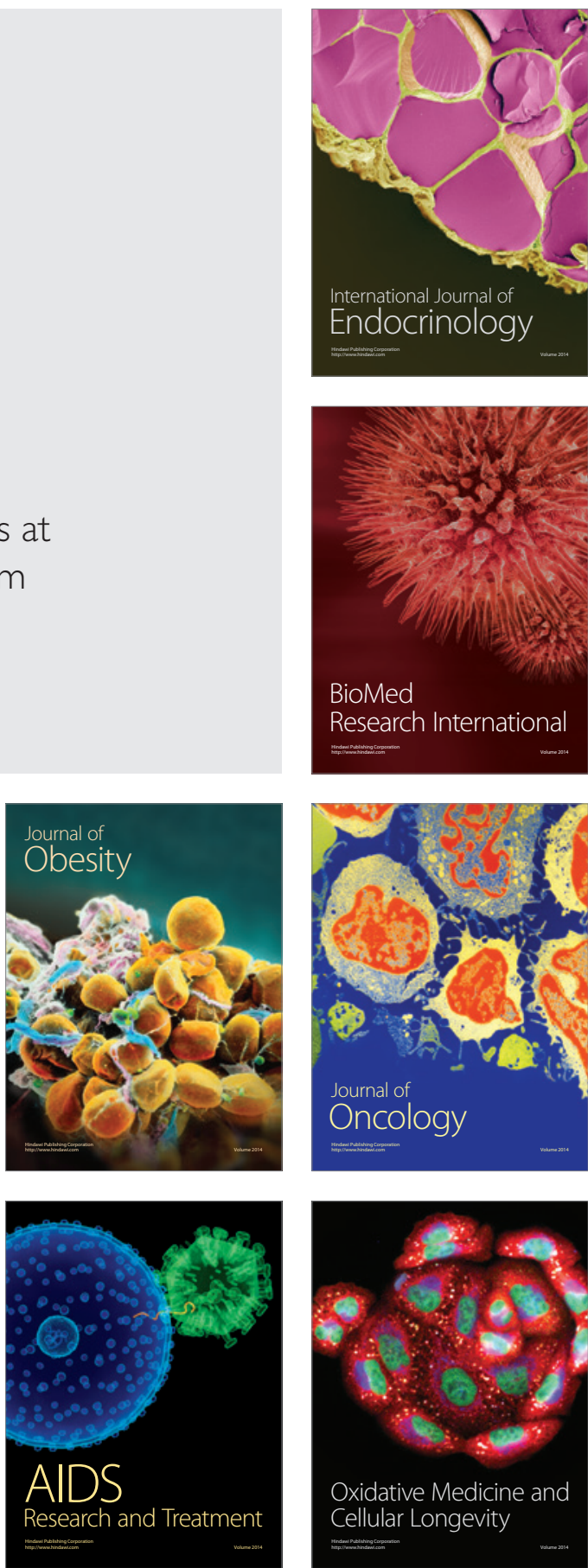